\title{
O EFEITO DA TAXA DE JUROS E DA \\ INCERTEZA SOBRE A CURVA DE PHILLIPS \\ DA ECONOMIA BRASILEIRA*
}

\author{
Ricardo de Oliveira Cavalcanti**
}

\section{Resumo}

Utilizando a hipótese de que as empresas brasileiras incorrem em custos financeiros ao contratar māo de obra, o trabalho sugere que a taxa de juros real esperada é um importante argumento da oferta agregada do país. Em adição, ao levar-se em conta que os empresários são a vessos ao risco, considerase que o coeficiente de variação da taxa de inflação também desloca a curva de Phillips. Os resultados empíricos revelam que a taxa de juros possui maior impacto do que a taxa de inflação sobre a curva de Phillips do Brasil.

\section{Introdução.}

Este trabalho visa estimar a curva de Phillips da Economia Brasileira, que acredita-se seja infiuenciada pela taxa real de juros esperada e pelo coeficiente de variação da taxa de inflação, além das variáveis que tradicionalmente são incorporadas pela literatura.

A taxa de juros afetaria o produto agregado porque as empresas incorrem num custo financeiro, ao adiantar o pagamento de seus insumos, com relação à data de recebimento do valor da venda dos produtos ${ }^{1}$. O coeficiente de variação da taxa de inflação, por ser um indicador da probabilidade dos produtores assumirem prejuizos, ao fecharem contratos indexados a índices de preços cuja realização desconhecem a priori, influi na decisão de produção, caso se admita alguma aversão ao risco por parte dos empresários.

*O autor agradece a Fernando de Holanda Barbosa, José Luiz Carvalho e Luiz Zottmann pelas valiosas críticas e sugestōes, e a Martha Spalenza Barcellos pela ajuda com o processamento dos dados. Os erros porventura remanescentes são de inteira responsabilidade do autor.

**Doutorando da EPGE/FGV.

${ }^{1}$ É freqüente, na indústria de transformação, a venda a prazo, sendo a folha salarial paga

a vista. A respeito da modelagem da curva de Phillips com taxa de juros, vide Barbosa (1989).

\begin{tabular}{llll}
\hline R. de Econometria Rio de Janeiro v. X, no 1, p.143-158 abril 1990 \\
\hline
\end{tabular}


O crescimento de ambas as variáveis citadas, por deslocar a demanda por mão de obra para a esquerda, tem efeito deletério sobre o desvio do produto, definido como a diferença entre os logaritmos do produto efetivo e potencial. O arrazoado da incorporação destas variáveis na curva de oferta agregada tem marco na tese de doutoramento de Domingo Cavallo², que procura investigar o processo inflacionário argentino, através de uma modelagem estruturalista.

No presente trabalho, contudo, a análise se concentrará na linha da sintese neoclássica-heynesiana de uma economia aberta com um só produto, cujo lado da oferta está representado pela relação de Phillips de preços, e o lado da demanda pelas curvas IS e LM. A derivação da curva de Phillips aqui utilizada, incorporando a taxa de juros e o coeficiente de variação da taxa de inflação, foi obtida por Cavalcanti (1990).

\section{O Modelo.}

As equações básicas do modelo log-linear são as seguintes:

$$
\begin{gathered}
y_{t}=\bar{y}_{t}+\beta\left(\pi_{t}-\pi_{t}^{*}\right)-\delta\left(r_{t}-\tilde{\pi}_{t+1}^{*}\right)-\xi \alpha_{\pi, t+1}^{2}+\alpha_{t} \\
y_{t}=D f_{t}-C\left(r_{t}-\tilde{\pi}_{t+1}^{*}\right)+\eta \theta_{t}+\nu_{t} \\
m_{t}-p_{t}=A y_{t}-\alpha r_{t}+v_{t}
\end{gathered}
$$

onde $\beta, \delta, \xi, D, C, \boldsymbol{\eta}, A$ e $\alpha$ são constantes positivas.

As variáveis $y_{t}, \bar{y}_{t}, \pi_{t}, f_{t}, r_{t}, \theta_{t}, m_{t}$ e $p_{t}$ representam, respectivamente, o produto efetivo, o produto quase potencial ${ }^{3}$, a taxa de inflação, o parâmetro de política fiscal, a taxa nominal de juros, a taxa real de câmbio, o agregado monetário $M 1$ e o índice de preços. O termo $\alpha_{\pi, t}^{2}$ é o coeficiente de variação ${ }^{4}$ da "taxa" de inflação, $1+\Pi_{t}$, especificada na forma composta ${ }^{5}$, que por sua vez

\footnotetext{
2Vide Cavallo (1977, 1991).

${ }^{3}$ O termo "quase" foi acrescentado ao potencial, com intençāo de guardar semelhança com o produto potencial tradicionalmente incorporado pela literatura. A respeito de defmiçóes alternativas de produto potencial vide McCallum (1980).

${ }^{4}$ Em verdade, $\alpha_{\pi, t}^{2}$ é o quadrado do coeficiente de variação. Continuaremos a chamá-lo apenas de coeficiente de variaçāo por simplicidade.

${ }^{5}$ Vale a seguinte relaçāo: $\pi_{t}=\log \left(1+\Pi_{2}\right)$.
}

\section{4}


$\therefore$ amida como tendo distribuição normal, com média $1+\Pi_{t}^{*}$, e variância $\alpha_{t}^{2}$.

As variáveis $\pi_{t}^{*}$ e $\tilde{\pi}_{t+1}^{*}$ representam as taxas esperadas de inflação, com base em informações adquiridas até o periodo $t-1$, para os períodos $t$ e $t+1$, respectivamente. Os erros $\varepsilon_{t}, \nu_{t}$ e $v_{t}$ são assumidos como sendo independentes, e possuindo média nula. Adicionalmente, supõe-se que $\varepsilon_{t}$ seja homocedástico e serialmente não correlacionado.

A equação (1) é a relação de Phillips de preços. Podemos t.omar a formulação tradicional da curva de Phillips como sendo um caso particular de (1), no caso em que $\delta=\xi=0$. Nesta sitnação não haveria custo de financiamento do capital de giro cas empresas, nem efeito de incerteza sobre o produto. Quando os agentes acertavam nas expectativas, obtendo-se $\pi_{t}=\pi_{t}^{*}$, a economia era dita encontrar-se em pleno emprego, acarretando $z_{t}=\bar{y}_{t}$.

No caso mais geral como indicado por (1), necessita-se de uma noção de pleno emprego que requer não apenas a igualdade $\pi_{t}=\pi_{t}^{*}$, como também que a taxa de juros real esperada $\left(r_{t}-\right.$ $\pi_{i+1}^{*}$ ) e o coefficiente de variação $\alpha_{\pi, t+1}^{2}$ estejam em seus niveis de equilibrio de longo prazo. Tais níveis, denotados por $\bar{\rho}$ e $\bar{\alpha}_{\pi}^{2}$, resprectivamente, são intrinsecos ao funcionamento da economia com estabilidade de taxas de inflação e um certo equilíbrio das contas do governo (por exemplo, déficit zero).

Supõe-se que os investimentos passados, que propiciaram a capacidade produtiva presente, foram orientados por uma perspectiva de lucratividade de longo prazo, parametrizada em $\bar{\rho}$ e $\bar{c}_{\pi}^{2}$. O produto de pleno emprego dos recursos da sociedade, deve então ser entendido como aquele que resulta do equilíbbrio do mercado de trabalho, $\bar{y}_{t}$, quando se tem $\pi_{t}=\pi_{t}^{*}$, descontando-se o efeito deletério do custo do capital de giro e da incerteza sobre as perspectivas dos empresários, dado por $\delta \bar{\rho}+\xi \bar{\alpha}_{\pi}^{2}$.

Se $\hat{y}_{t}=\bar{y}_{t}-\delta \bar{\rho}-\xi \bar{\alpha}_{\pi}^{2}$ é a definição de produto de pleno emprego adequada, então a equação (1) pode ser reescrita na forma seguinte:

$$
y_{t}=\hat{y}_{t}+\beta\left(\pi_{t}-\pi_{t}^{*}\right)-\delta\left[\left(r_{t}-\tilde{\pi}_{t+1}^{*}\right)-\bar{\rho}\right]-\xi\left(\alpha_{\pi, t+1}^{2}-\bar{\alpha}_{\pi}^{2}\right)+\varepsilon_{t}
$$

Como observa-se em (1'), o produto quase potencial $\bar{y}_{t}$ corresponde ao produto de pleno emprego, $\hat{y}_{t}$, no caso de $\delta \bar{\rho}+\xi \bar{\alpha}_{\pi}^{2}$ ser igual a zero. 
Também está implícito, na presente formulação da curva de Phillips, que foi desprezado o efeito dos preços de insumos importados sobre os custos da produção agregada. Contudo, o setor externo foi considerado no lado da demanda.

A equação (2) representa a curva $I S$, de equilíbrio do mercado de bens. O saldo das exportações sobre importações de não fatores é função da taxa real de câmbio $\theta_{t}$, além de também ser determinado pelo nivel de renda. A variável $f_{t}$ captura o efeito dos gastos do governo, além dos gastos privados autônomos, sobre o intercepto da $I S$.

A curva $L M$, de equilíbrio do mercado monetário, descrita pela equação (3), foi obtida através da hipótese usual de que a demanda por $M 1$ é função do nivel de renda real e da taxa nominal de juros.

Com relação à lei de formação de expectativas, foi considerado que os agentes econômicos utilizam uma combinação linear das taxas de inflação passadas, para preverem a taxa de inflação do periodo corrente. Os pesos da combinação linear, ao longo do periodo abrangido, somam um. Os agentes possuem o grau de liberdade de utilizar em $\pi_{t}^{*}$ pesos distintos daqueles designados para obter $\tilde{\pi}_{t+1}^{*}$.

A lei de formação de expectativas, como descrita acima, abrange os casos particulares de expectativas estáticas, aceleracionistas e adaptativas. Entretanto, como não se conhece a priori a combinação linear utilizada para prever $\pi_{t}$. ou sua expressão na forma composta $\Pi_{t}$, introduz-se um termo não linear na equação (1). Com efeito, uma vez que $\alpha_{\pi, t}^{2}=\alpha_{t}^{2} /\left(1+\Pi_{t}^{*}\right)^{2}$ torna-se uma função não linear das defasagens de $\pi_{t}$, o produto efetivo passa a depender das taxas de inflação passadas de forma não linear.

Neste trabalho, optou-se por adotar $\alpha_{t}^{2} /\left(1+\Pi_{t}\right)^{2}$ como proxy de $\alpha_{\pi, t}^{2}$, decisão esta que se deve a dois fatos básicos. O primeiro é que $\alpha_{t}^{2}$ é uma variávei não observável, constituindo-se numa apreciação subjetiva que os produtores atribuem ser a variância da taxa de inflação. Neste quadro, se espera que os erros de medida introduzíveis não comprometam a significância do sinal do efeito da variável de incerteza sobre o produto.

O segundo fato diz respeito a facilidades computacionais. Acredita-se que os ganhos advindos de uma forma funcional não linear tornam-se desprezíveis, uma vez que substituir $\Pi_{t}^{*}$ por $\Pi_{t}$ 
em $\alpha_{\pi, t}^{2}$ nãoão altera o sinal do coeficiente de variação da taxa de inflação, apesar de introduzir algum viés.

\section{A Identificaçāo da curva de Phillips.}

Com o objetivo de discutir o problema de identificação da curva de Phillips, inserida no sistema básico de equações dado por

(1'), (2) e (3), torna-se necessário apresentar o modelo completo, como segue:

$$
\begin{aligned}
h_{t}= & \sum_{j}^{k} \delta_{j} d_{j t}+\sum_{i}^{n} \lambda_{i} h_{t-i}+\beta_{11} c+\beta_{12} \pi_{t}+\beta_{13} \pi_{t}^{*} \\
& +\beta_{14} r_{t}+\beta_{15} \tilde{\pi}_{t+1}^{*}+\sum_{i}^{n} \theta_{i} a_{\pi, t-i}^{2}+\varepsilon_{1 t} \\
y_{t} & =\beta_{21} c+\beta_{24} r_{t}+\beta_{25} \tilde{\pi}_{t+1}^{*}+\beta_{26} f_{t}+\beta_{27} \theta_{t}+\varepsilon_{2 t} \\
y_{t} & =\beta_{31} c+\beta_{34} r_{t}+\beta_{38} m_{t}+\beta_{3,9} p_{t}+\varepsilon_{3 t} \\
h_{t} & =y_{t}-\hat{y}_{t} \\
\hat{y}_{t} & =\bar{y}_{t}+\beta_{51} c \\
\bar{y}_{t} & =\beta_{61} c+\beta_{6.10} T_{t}^{t}+\varepsilon_{6 t} \\
\pi_{t} & =p_{t}-p_{t-1} \\
\pi_{t}^{*} & =\sum_{0}^{n} \pi_{t-i} \\
\tilde{\pi}_{i+1}^{*} & =\sum_{i}^{n} \omega_{i} \pi_{t-i}
\end{aligned}
$$

Os termos $c, \boldsymbol{\alpha}_{j t}$ e $T_{t}$ representam, respectivamente, intercepto, dummies sazonais e variável de tendência.- As demais variáveis mantém o mesmo significado das seções anteriores.

A equação (1a) é a relação de Phillips de preços, obtida a partir de (1'), especificada em termos do desvio do produto $h_{t}$, definido em (4a). A constante $c$ absorve os termos fixos do produto de pleno emprego. Foram acrescentadas defasagens dos desvios do produto, de modo a captar inércia no ajustamento da produção, defasagens do coeficiente de variação da taxa de inflação, devido à 
distribuição dos efeitos de incerteza ao longo do tempo, e dummies destinadas a captar sazonalidade.

As curvas $I S$ e $L M$ estão representadas por (2a) e (3a). As equações (5a) e (6a) tratam dos produtos de pleno emprego e quase potencial. O termo $T_{t}$ foi tulizado para captar o crescimento da capacidade produtiva, que supõe-se ocorrer a uma taxa constante. As equações (7a), (8a) e (9a) definem, respectivamente, a taxa de inflação e as leis de formação de expectativas para os periodos $t$ e $t+1$.

Substituindo-se as identidades (4a), (5a), (8a) e (9a), além da equação (6a), nas equações restantes, obtém-se o seguinte:

$$
\begin{gathered}
h_{t}=\sum_{j}^{k} \delta_{j} \alpha_{j t}+\sum_{i}^{n} \lambda_{i} h_{t-i}+\beta_{11} c+\beta_{12} \pi_{t} \\
+\sum_{i}^{n} \gamma_{i} \pi_{t-i}+\beta_{14} r_{t}+\sum_{i}^{n} \theta_{i} \alpha_{\pi, t-i}^{2}+\varepsilon_{1 t} \\
h_{t}=\hat{\beta}_{21} c-\beta_{6,10} T_{t}+\beta_{24} r_{t}+\sum_{i}^{n} \hat{\omega}_{i} \pi_{t-i} \\
+\beta_{26} f_{t}+\beta_{27} \theta_{t}+\hat{\varepsilon}_{2 t} \\
h_{t}=\hat{\beta}_{31} c-\beta_{6,10} T_{t}+\beta_{34} r_{t}+\beta_{39} m_{t}+\beta_{3,9} p_{t}+\hat{\varepsilon}_{3 t} \\
0=-\pi_{t}+p_{t}-p_{t-1}
\end{gathered}
$$

onde,

$$
\begin{aligned}
\gamma_{i} & =\beta_{13} \alpha_{i}+\beta_{15} \omega_{i} \\
\hat{\beta}_{21} & =\beta_{21}-\beta_{51}^{\prime}-\beta_{61} \\
\hat{\beta}_{31} & =\beta_{31}-\beta_{51}-\beta_{61} \\
\hat{\omega} & =\beta_{25} \omega_{i} \\
\hat{\varepsilon}_{2 t} & =\varepsilon_{2 t}-\varepsilon_{67} \\
\hat{\varepsilon}_{3 t} & =\varepsilon_{3 t}-\varepsilon_{67}
\end{aligned}
$$

Escrevendo o sistema de equações dado por (1b), (2b), (3b) e (7a) em forma matricial, ob tém-se a seguinte matriz $A_{[4 \times(10+k+0 n)]}$ dos parâmetros da forma estrutural ${ }^{6}$ :

${ }^{6}$ Em verdade, $A$ é uma matriz de parâmetros de uma forma semi-reduzida. 


$$
\begin{aligned}
& A= \\
& {\left[\begin{array}{cccccccccccccc}
-1 & \vec{\delta} & \tilde{\lambda} & \beta_{11} & \vec{\gamma} & \beta_{14} \vec{s} & 0 & 0 & 0 & 0 & 0 & 0 & & \\
-1 & 0 & 0 & \beta_{21} & 0 & \vec{\omega} & \beta_{24} & 0 & -\beta_{6,10} & \beta_{26} & \beta_{27} & 0 & 0 & 0 \\
-1 & 0 & 0 & \varepsilon_{31} & 0 & 0 & \beta_{34} & 0 & -\beta_{6,10} & 0 & 0 & \beta_{38} & \sigma_{39} & 0 \\
\bullet & 0 & 0 & 0 & -1 & 0 & 0 & 0 & 0 & 0 & 0 & 0 & 1 & -1
\end{array}\right]}
\end{aligned}
$$

onde $\vec{\delta}, \vec{\lambda}, \vec{\gamma}$ e $\vec{s}$ são vetores que reunem os termos $\delta_{j}, \lambda_{i}, \gamma_{i}$ e $s_{i}$.

As restrições de exclusão de variáveis que incidem sobre a primeira equação podem ser expressas por uma matriz $R_{[(10+k+3 n) \times 6] \text {, }}$ tal que

$$
\left[\begin{array}{c}
0 \\
\cdots \\
I_{6}
\end{array}\right]
$$

ou seja, $R$ pode ser partida entre uma matriz nula e uma matriz identidade de sexta ordem.

A primeira equação é identificada se a matriz $A R_{(4 \times 6)}$, produto entre $A$ e $R$, for de posto três. Á matriz $A R$ é dada por:

$$
A R=\left[\begin{array}{cccccc}
0 & 0 & 0 & 0 & 0 & 0 \\
-\beta_{6,10} & \beta_{26} & \beta_{27} & 0 & 0 & 0 \\
-\beta_{6,10} & 0 & 0 & \beta_{38} & \beta_{33} & 0 \\
0 & 0 & 0 & 0 & 1 & -1
\end{array}\right]
$$

Como as três últimas linhas de $A R$ são linearmente independentes (a menos que os coeficientes envolvidos sejam nulos). pode-se afirmar que esta matriz é posto três. Desie modo, sob as restrições de exclusão de variávcis, a primeira equação é identificada.

Uma vez que o modelo, como apresentado pela matriz $A$, impõe seis restrições de exclusão de variáveis sobre a primeira equação, o grau de mais que identificação da curva de Phillips á três.

As variáveis endógenas do modelo são $h_{t}, \pi_{t}, r_{t}$ e $p_{t}$.

De modo a simplificar o processo de estimação, não fo: imposto, a priori, uma importante restrição sobre a primeira equação. Esta restrição, decorrente do fato de que é desejável que uma curva de oferta seja homogênea do grau zero nos preços de fatores e produto, impõe que tanto os coeficientes de $\pi_{t}$ e $\pi_{i}^{*}$, 
como os de $r_{t}$ e $\tilde{\pi}_{t+1}^{*}$, devem ser simétricos entre si. Como apresentado pela matriz $A$, o modelo precisa então satisfazer a seguinte restrição homogênea:

$$
\beta_{12}+\sum_{i} \gamma_{i}+\beta_{14}=0
$$

Como se verá na seção seguinte, os coeficientes estimados da curva de Phillips do Brasil satisfazem tal restrição.

\section{Metodologia e Resultados.}

O método utilizado para estimar a curva de Phillips da economia brasileira foi o de mínimo quadrados de dois estágios. No primeiro estágio foram feitas duas regressões, com mínimos quadrados ordinários.

A primeira regressão utilizou a taxa de juros $r_{t}$ como variável dependente, e as variáveis predeterminadas do modelo completo como independentes; ou seja, se estimou a equação da forma reduzida de $r_{t}$. Como $P_{t}$ também é função das mesmas variáveis predeterminadas, então pela equação ( $7 a), \pi_{t}$ é função de suas primeiras diferenças. Desta feita, a sgunda regressão foi a estimação da forma reduzida de $\pi_{t}$, em termos das primeiras diferenças das variáveis predeterminadas.

De posse dos valores ajustados de $\pi_{t}$ e $r_{t}$, obtidos nas primeiras regressões, o sgundo estágio tratou de estimar a equação (1b), substituindo estes valores no lugar das variá veis originais, e aplicando novamente mínimos quadrados ordinários.

Trabalhando-se com séries trimestrais, procurou-se abranger - maior período possível, dentro da disponibilidade de dados macroeconômicos do Brasil no momento da elaboração deste trabalho: segundo trimestre de 1976 até o primeiro trimestre de 1989 .

A seguir tem-se a lista das variáveis, com suas respectivas descrições e fontes.

$h_{\ell}$ : Média trimestral do logaritmo da taxa de utilização da capacidade instalada, da indústria de transformação, calculada pela FIESP e obtida através do projeto ARIES-FGV. Foram somados vinte pontos percentuais à série original, de modo a fazer o percentual médio de ocupação ao longo do período, cerca de $80 \%$, corresponder à taxa de utilização de pleno emprego. 
$\pi_{t}$ : Primeira diferença trimestral do logaritmo do IGP-DI, calculado pela FGV e obtido através do projeto ARIES-FGV.

$r_{t}$ : Média trimestral da taxa logarítmica mensal, baseada na taxa de desconto de duplicatas de bancos comerciais, publicada pela revista Exame.

$\alpha_{\pi, t}^{2}$ : Baseado nas dez desagregações do IPA-DI publicadas pela Conjuntura Econômica, e obtidas junto ao projeto ARIESFGV. Denominando de $\Pi_{i, t}$ a taxa composta mensal de inflação baseada na desagregação $i$, e de $\mu_{t}$ a média dos $\Pi_{i, t}$ 's, então o valor mensal do coeficiente de variação, $\alpha_{\pi, t}^{2}$, é dado por:

$$
\alpha_{\pi, t}^{2 *}=\frac{1}{\left(1+\mu_{t}\right)^{2}} \sum_{i=1}^{9}\left(\Pi_{i, t}-\mu_{t}\right)^{2} / 9 .
$$

A variável $\alpha_{\pi, t}^{2}$ é a média trimestral de $\alpha_{\pi, t}^{2 *}$.

$f_{t}$ : Primeira diferença da média trimestral do logaritmo do Total da Dívida Federal (deflacionado pelo IGP-DI), publicado pela Conjuntura Econômicà e obtido através do projeto ARIESFGV.

$\theta_{t}$ : Logaritmo da taxa de câmbio real, baseada no preço de venda do Dólar e nos IPA's do Brasil e dos Estados Unidos. A série foi obtida no projeto ARIES-FGV, a partir de 1978, e complementada com informações do IFS-FMI.

$m_{t}$ : Média trimestral do logaritmo do agregado monetário $M 1$, obtido através do projeto ARIES-FGV.

$d_{2 t}, d_{3 t}, d_{4 t}$ : Dummies sazonais que assumem valor um nos segundos, terceiros e quartos trimestres, respectivamente.

$d_{5 t}$ : Assume valor um no sgundo, terceiro e quarto trimestre de 1986, e valor zero nos demais periodos.

$d_{6 t}$ : Assume valor um a partir do segundo trimestre de $198 \dot{6}$, e valor zero nos periodos anteriores.

$T_{\imath}$ : Variável que aumenta de uma unidade a cada periodo.

$c$ : Assume valor um em todos os periodos.

$\boldsymbol{p}_{t}$ : Logaritmo do IGP-DI.

Alguns comentários sobre as séries de dados utlizadas permitem precisar melhor os resultados obtidos nas regressões.

O primeiro ponto importante diz respeito à medição que está sendo utilizada do desvio do produto. Uma vez que a série de utilização da capacidade instalada se restringe à indústria de trans- 
formação, os resultados obtidos devem ser contingenciados ao peso deste setor na produção agregada do país.

O coeficiente de variação, que é baseado em quatro taxas de inflação de bens de consumo, e seis de bens de produção, reflete a dispersão destas taxas em torno de um valor médio. Se os agentes acreditam que a taxa de inflação relevante é uma média ponderada das demais, então o coeficiente de variação será uma boa aproximação da medida equivalente que os agentes têm em mente, caso seus cálculos sejam baseados na evolução dos preços de grandes setores, como aqueles distinguidos na desagregação do IPA-DI.

Quanto aos sinais esperados dos coeficientes da equação ( $1 \mathrm{~b}$ ), apesar da apresentação do modelo ocorrida até o momento ter esgotado este ponto, vale a pena chamar a atenção para dois aspectos.

O primeiro é que as defasagens de $\pi_{t}$, que entram no modelo através das definições de $\pi_{t}^{*}$ e $\tilde{\pi}_{t+1}^{*}$, podem ter coeficientes com qualquer sinal, como se observa através da construção dos coeficientes $\gamma_{i}$ 's.

O segundo aspecto é que se espera uma contribuição positiva de $d_{5 t}$, devido aos choques de oferta positivos que ocorreram durante o Plano Cruzado, possibilitando redução de custni, em razão, por exemplo, da contenção de tarifas públicas ocorrida em 1986. A variável $d_{6 t}$ procura captar um possivel deslocamenteo da curva de Phillips, decorrente da introdução de expectativas de congelamentos de preços que pudessem surpreender os empresários, e que passaram a fazer parte do horizonte dos produtores a partir do primeiro congelamento.

Os resultados da estimação da curva de Phillips para a economia brasileira, encontram-se na Tabela 1.

Tanto na Tabela 1 quanto nas Tabelas 2,3 e 5 (apresentadas abaixo), $R^{2}$ é o coeficiente de determinação, $\bar{R}^{2}$ é o coeficiente corrigido, $S Q R$ é o somatório dos quadrados dos resíduos, $F$ é a estatística " $F$ " do teste da nulidade conjunta dos coeficientes, $D P R$ é o desvio padrão da regressão, $D W$ é a estatística de Durbin-Watson para se testar autocorrelação de primeira ordem dos resíduos, $C S$ é a estatística $L M$ para se testar autocorrelação de quarta ordem dos resíduos, $F F$ é a estatística do RESET de Ramsey para se testar forma funcional, $N$ é a estatística de Bera-Jarque para se testar normalidade dos resíduos; e $H E$ é a estatística para se testar heterocedasticidade dos risíduos.

\section{2}




\section{Tabela 1.}

Estimação de $(1 \mathrm{~b})^{7}$

(Variável dependente: $h_{t}$ )

\begin{tabular}{lccccc}
\hline Variáveis: & $d_{2 t}$ & $d_{\partial t}$ & \multicolumn{1}{c}{$d_{47}$} & $d_{5 t}$ & $d_{6 t}$ \\
Estimativas: & .0278 & .0184 & .0093 & .0308 & -.0362 \\
Estat. "T": & 5.6965 & 4.0545 & 2.1554 & 3.4297 & -2.8947 \\
$\ldots \ldots$ & $\ldots \ldots$ & $\ldots \ldots$ & $\ldots \ldots$ & $\ldots \ldots$ & $\ldots \ldots$ \\
Variáveis: & $h_{t-1}$ & $h_{t-3}$ & $\hat{r}_{t}$ & $\hat{\pi}_{t}$ & $\pi_{t-2}$ \\
Estimativas: & 1.0837 & -.2712 & -.1196 & .0595 & -.0387 \\
Estat. "T": & 10.9311 & -2.7966 & -3.6073 & 2.2419 & -1.9731 \\
$\ldots . .$. & $\ldots \ldots$ & $\ldots \ldots$ & $\ldots \ldots$ & $\ldots \ldots$ & $\ldots \ldots$ \\
Variáveis: & $\pi_{t-8}$ & $\pi_{t-13}$ & $\pi_{t-14}$ & $\sigma_{\pi, t}^{2}$ & $\sigma_{\pi, t-2}^{2}$ \\
Estimativas: & .0671 & -.0911 & .1107 & -.6114 & -.6853 \\
Estat. "T": & 2.7185 & -1.6975 & 2.1432 & -2.0734 & -2.8944 \\
$\ldots . .$. & $\ldots \ldots$ & $\ldots \ldots$ & $\ldots \ldots$ & $\ldots \ldots$ & $\ldots \ldots$ \\
& $R^{2}: .9507$ & $\bar{R}^{2}: .9321$ & $S Q R: .0037$ & $F^{a}: 50.9953$ & $D P R: .0100$ \\
& $D W: 1.9049$ & $C S^{b}: .8395$ & $F F^{c}: .5972$ & $N^{d}: .6140$ & $H E^{e}: .0975$ \\
\hline
\end{tabular}

Distribuição das estatísticas:
(a) $F(14,37)$.
(b) $F(4,33)$. (c) $F(1,36)$.
(d) $Q(2)$. (e) $F(1,50)$.

tatística para se testar heterocedasticidade dos residuos.

As estimativas da Tabela 1 já são resultado de um processo de seleção das variáveis significantes. Apenas duas defasagens da taxa de inflação apresentaram estatística $T$ maior do que dois. Este fato é explicado pelo modelo devido aos sinais contrários que são esperados para os coeficientes de $\pi_{t}^{*}$ e $\tilde{\pi}_{t+1}^{*}$. Quanto ao desvio do produto, apenas duas defasagens são significantes. As estimativas dos coeficientes de $h_{t-1}$ e $h_{t-3}$ revelam uma equação de diferenças finitas, no desvio do produto, estável.

O coeficiente de variação do período $t+1$ não foi significante, passando a constar da seleção de variáveis apenas aqueles referentes aos periodos $t$ e $t-2$. Este evento revela uma certa inércia do efeito da incerteza sobre o produto, talvez justificada por defasagens entre o tempo da tomada de decisão de iniciar a produção, e o tempo de iniciar a produção em si.

Os sinais de todos os coeficientes foram aqueles esperados, como postula o modelo. Destacam-se, a propósito, a elevada signı- 
ficância da taxa de juros, e o fato do coeficiente desta variável ser maior, em módulo, do que aquele relativo à taxa de inflação do período $t$, que faz parte das formulações tradicionais da curva de Phillips de preços como variável mais importante.

As estatísticas apresentadas na Tabela 1 garantem a consistência do modelo. As estatísticas $C S, F F, N$ e $H E$ corroboram as hipóteses até aqui adotadas. Não se pode rejeitar as hipoteses de que os erros são normais, homocedásticos e serialmente não correlacionados, e que a forma funcional, a um nivel de significância de $5 \%$, está correta.

A Tabela 2 aprsenta a regressão referente à taxa de juros, enquanto a Tabela 3 o faz para a taxa de inflação. Nesta última, se utilizou a letra " $L$ ", antes dos símbolos já apresentados, para denotar que se trata da primeira diferença da variável. Note que a primeira diferença de $T_{t}\left(L T_{t}\right)$ é identicamente igual à constante c.

Ainda com relação à regressão da curva de Phillips, resta verificar se a restrição (5) é satisfeita pelos coeficientes da equação (1b). Este teste pode ser feito calculando-se um intervalo de confiança para a função linear, dada por (5), sobre o vetor de coeficientes de $(1 b)^{8}$. Se $b$ representa este vetor, então a restrição (5) pode ser reescrita como:

$$
w^{\prime} b=0
$$

onde $w$ é um vetor que assume valor um nas coordenadas referentes aos coeficientes citados em (5), e zero nas demais. Se $\Xi$ é a matriz de variância-covariância de $b$, então $\left(w^{\prime} b\right) / \sqrt{w^{\prime} \Xi w}$ é assintoticamente normalmente distribuida. O termo $\boldsymbol{w}^{\prime} \Xi \boldsymbol{w}$ representa o somatório de um conjunto particular de covariâncias, associadas aos coeficientes citados em (5), relacionadas numa sub-matriz de $\Xi$. Os valores estimados desta sub-matriz encontram-se na Tabela 4.

Com base nas Tabelas 1 e 4, verifica-se que a estatistica para o teste da restrição (5) assume, em módulo, o valor 0,3005. Logo, a um nivel de significância de $5 \%$, a equação (1b) satisfaz a restrição de ser homogênea do grau zero nos preços.

Finalmente, os valores indicados na Tabela 1 sugerem que se deva utilizar a primeira diferença do desvio do produto $\left(L h_{t}\right)$ como variável dependente na curva de Phillips, uma vez que a

${ }^{8}$ A este respeito, vide Theil (1971), p. 131-139.

\section{4}




\section{Tabela 2.}

Estimaçāo da $1 \underline{a}$ forma reduzida

(Variável dependente: $r_{t}$ )

\begin{tabular}{|c|c|c|c|c|c|}
\hline Variáveis: & $c$ & $d_{2 t}$ & $\delta_{97}$ & $\delta_{4 t}$ & $\delta_{5 t}$ \\
\hline Estimativas: & .7991 & -.0099 & -.0310 & -.0098 & -.1197 \\
\hline Estat. "T": & 1.4526 & -.2946 & -.9086 & -.2660 & -.7707 \\
\hline Variáveis: & $\delta_{b t}$ & $\underset{h_{t-1}}{\cdots \cdots}$ & $\underset{h_{t-3}}{\cdots \cdots}$ & $m_{t}$ & $\ddot{f}_{t}$ \\
\hline Estimativas: & -.2356 & .2474 & -1.0353 & .0000 & .1151 \\
\hline Estat. "T": & -2.3207 & .3937 & -1.9326 & 2.0535 & .6971 \\
\hline Variáveis: & $\hat{\theta}_{t}^{\cdots \cdots}$ & $\tilde{T}^{\cdots \cdots}$ & $\sigma_{\pi, t}^{2} \cdots \cdots$ & $\sigma_{\pi, t-2}^{2} \cdots \cdots$ & $p_{t-1}^{2}$ \\
\hline Estimativas: & -.1809 & .0044 & 1.0846 & 2.5847 & -.0006 \\
\hline Estat. "T": & -1.3766 & 1.3399 & .4948 & 1.2441 & -1.9166 \\
\hline$\because \cdots \cdots$ & $\cdots \cdots$ & .... & $\ldots \ldots$ & $\ldots \ldots$ & $\ldots \ldots$ \\
\hline Variáveis: & $\pi_{t-1}$ & $\pi_{t-2}$ & $\pi_{t-3}$ & $r_{t-4}$ & $\pi_{t-5}$ \\
\hline Estimativas: & .1296 & .2011 & -.2230 & -.0064 & .4721 \\
\hline Estat. "T": & .7915 & .9704 & -1.3827 & -.0307 & 2.3359 \\
\hline$\cdots$ & $\ldots \ldots$ & $\ldots \ldots$ & $\ldots \ldots$ & $\ldots \ldots$ & $\ldots \ldots$ \\
\hline Variáveis: & $\pi_{t-6}$ & $\pi_{t-7}$ & $\pi_{t-8}$ & $\pi_{t-9}$ & $\pi_{t-10}$ \\
\hline Estimativas: & -.1788 & -.4706 & .4571 & -.0428 & .3639 \\
\hline Estat. "T": & -.6662 & -1.5983 & 1.5545 & -.1594 & 1.2810 \\
\hline$\ldots \ldots$ & $\ldots \ldots$ & $\ldots \ldots$ & $\cdots \cdots$ & ..... & $\ldots \ldots$ \\
\hline Variáveis: & $\pi_{t-11}$ & $\pi_{t-12}$ & $\pi_{t-13}$ & $\pi_{t-14}$ & \\
\hline Estimativas: & .0052 & -.2832 & -.2819 & -.0534 & \\
\hline Estat. "T": & .0161 & .9068 & -.8462 & -.1280 & \\
\hline \multirow[t]{3}{*}{$\ldots \ldots$} & $\ldots \ldots$ & $\ldots \ldots$ & $\ldots$ & $\ldots \ldots$ & $\ldots \ldots$ \\
\hline & $R^{2}: .9507$ & $\bar{R}^{2}: .9347$ & $S Q R: .0395$ & $F^{a}: 27.0563$ & $D P R: .0415$ \\
\hline & $D W: 2.1745$ & $C S^{b}: 1.7063$ & $F F^{c}: 3.5750$ & $N^{d}: 7.7280$ & $H E^{e}: .9262$ \\
\hline
\end{tabular}

Distribuição das estatísticas:

(a) $F(28,23)$. (b) $F(4,19)$. (c) $F(1,22)$. (d) $Q(2)$. (e) $F(1,50)$.

estimativa do coeficiente de $\boldsymbol{h}_{t-1}$ é aproximadamente igual a um. Esta sugestão é levada a cabo na Tabela 5. Apesar dos valores das estimativas não se modificarem muito, a elevação da estatística referente ao teste da forma funcional $(F F)$, revela que talvez esta não seja a forma mais indicada para a curva de Phillips.

\section{Conclusōes.}

As estimativas ora aprsentadas para a curva de Phillips de preços do Brasil não permitem rejeitar a hipótese de que tanto a taxa real de juros esperada, quanto o coeficiente de variação de 
Tabela 3.

Estimaçāo da $2 \stackrel{\text { a }}{\text { forma reduzida }}$

(Variável dependente: $\pi_{t}$ )

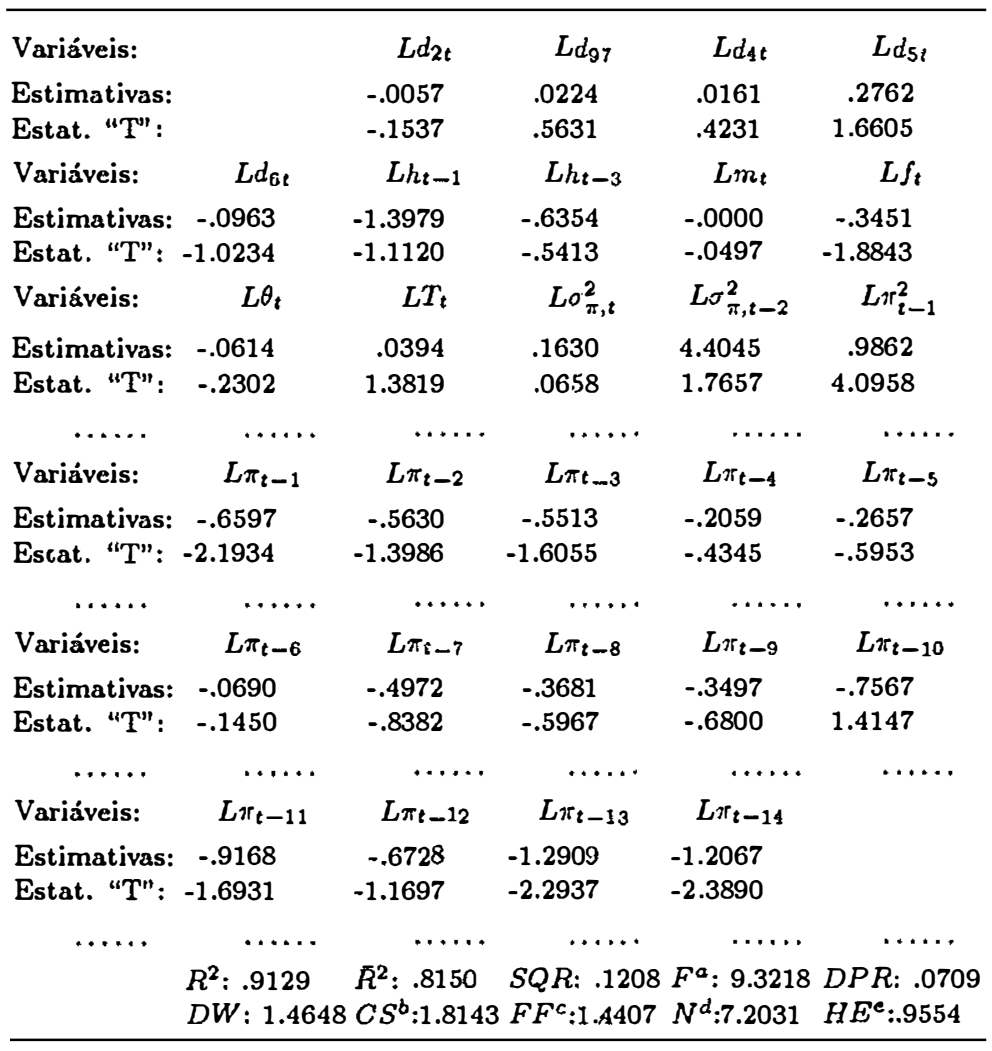

Distribuiçāo das estatísticas:

(a) $F(27,24)$. (b) $F(4,20)$. (c) $F(1,23)$. (d) $Q(2)$. (e) $F(1,50)$.

taxa de inflação, afetam significativamente o desvio do produto. Com este trabalho pretende-se jogar luz sobre a importância da administração de políticas macroeconômicas que evitem incertezas quanto ao nível de preços da economia, e que visem a manutenção, no longo prazo, da taxa de juros em certo equilíbrio, como forma de obtenção do produto de pleno emprego.

Por outro lado, os dados parecem indicar uma baixa elasti-

\section{6}


Tabela 4.

Matriz de variância-covatiância dos paramêtros de $(1 \mathrm{~b})^{9}$

\begin{tabular}{rrrrrrr}
\hline & \multicolumn{1}{c}{$\hat{r}_{t}$} & \multicolumn{1}{c}{$\hat{\pi}_{t}$} & $\pi_{t-2}$ & $\pi_{t-9}$ & $\pi_{t-19}$ & $\pi_{t-14}$ \\
& & & & & & \\
$\hat{r}_{t}$ & .0011 & -.0006 & -.0000 & -.0004 & .0001 & -.0004 \\
$\hat{\pi}_{t}$ & -.0006 & .0007 & -.0000 & .0003 & -.0001 & -.0001 \\
$\pi_{t-2}$ & -.0000 & -.0000 & .0003 & -.0000 & -.0003 & .0000 \\
$\pi_{t-9}$ & -.0004 & .0003 & -.0000 & .0006 & -.0003 & -.0000 \\
$\pi_{t-13}$ & .0001 & -.0001 & -.0003 & -.0003 & .0028 & -.0013 \\
$\pi_{t-14}$ & -.0004 & -.0001 & .0000 & -.0000 & -.0023 & .0002 \\
\hline
\end{tabular}

$\left(^{*}\right)$ Contempla apenas os coeficientes que sofrem a restrição (5).

Tabela 5.

Estimaçāo de (1b) ${ }^{10}$

(Variável dependente: $L h_{t}$ )

\begin{tabular}{|c|c|c|c|c|c|}
\hline Variáveis: & $d_{2 t}$ & $d_{\delta t}$ & $d \notin 7$ & $d_{5 t}$ & $d_{\text {it }}$ \\
\hline Estimativas: & -.0272 & .0185 & .0105 & .0317 & -.0337 \\
\hline Estat. "T": & 5.6551 & 4.1098 & 2.5954 & 3.5652 & -2.7841 \\
\hline$\ldots \ldots$ & $\cdots \cdots$ & $\cdots \cdots$ & $\ldots \ldots$ & $\cdots \cdots$ & $\ldots \ldots$ \\
\hline Variáveis: & & $h_{t-3}$ & $\hat{r}_{t}$ & $\dot{\pi}_{t}$ & $\pi_{t-2}$ \\
\hline Estimativas: & & -.2008 & -.1175 & -.1175 & -.0390 \\
\hline Estat. "T": & & -4.0837 & -4.0837 & -3.5660 & -1.9936 \\
\hline$\ldots \cdots$ & $\cdots \cdots$ & $\cdots \cdots$ & $\ldots \cdots$ & $\ldots+\cdots$ & $\ldots \ldots$ \\
\hline Variáveis: & $\theta_{t-8}$ & $\pi_{t-13}$ & $\pi_{t-14}$ & $\sigma_{\pi, t}^{2}$ & $\sigma_{\pi, t-2}^{2}$ \\
\hline Estimativas: & -.0642 & -.0990 & .1112 & -.5694 & $-\quad-.6752$ \\
\hline Estat. "T": & 2.6372 & -1.8812 & 2.1609 & -1.9665 & -2.8663 \\
\hline \multirow[t]{3}{*}{$\ldots \cdots$} & $\ldots \ldots$ & $\ldots \ldots$ & $\ldots \ldots$ & $\cdots \cdots$ & $\ldots \ldots$ \\
\hline & $R^{2}: .6032$ & $\bar{R}^{2}: .4 \$ 74$ & $S Q R: .0038$ & $F^{a}: 4.4428$ & $D P R: .010$ \\
\hline & $D W: 1.7357$ & $C S^{b}: 3997$ & $F F^{c}: 3.3987$ & $N^{d}: .7271$ & $H E^{e}: .8273$ \\
\hline
\end{tabular}

Distribuição das estatísticas:
(a) $F(13,38)$.
(b) $F(4,34)$. (c) $F(1,37)$.
(d) $Q(2)$. (c) $F(1,50)$. 
cidade-preço da oferta agregada. Uma possivel explicação para este fenômeno, que se traduz em uma curva de Phillips aproximadamente vertical, reside na contrapartida do recrudescimento inflacionário, acompanhado de crescente volatilidade de preços, como ocorreu no País na década de 80 . A hipótese, ainda por ser testada, seria de que a inconsistência da condução de politica econômica no período acabou por dificultar consideravelmente a extração dos sinais dos preços relativos. Em conformidade com o mołelo de Lucas (1977), a crescente introdução de incerteza terminaria por reduzir a zero a elasticidade da curva de Phillips do Brasil.

\section{Referências}

Barbosa, F. de H. Macroeconomia. Ensaios Econômicos n* 139, EPGE/FGV. 1989.

Cavalcanti, R. de O. Inflação, estagnação e incerteza: teoria e experiência brasileira. Dissertação de mestrado EPGE/FGV.

Cavallo, D.F. Stagflationary eflects of monetarist stabilization polices in economies with persistent inflation. Ph.D. Thesis. Harvard University.

Stagflationary effects of monetarist stabiliztion polices in economies with persitent inflation. Academic Press, Inc.. In Development in an Inflationary World, 1981.

Lucas, R. E. Jr. Some international evidence on output-inflation tradeoffs. American Economic Review, 63:1973.

McCallum, B.T. Rational expectations and macroeconomic stabilization policy. Journal of Money, Credit and Banking, November 1980, Part 2.

Theil, H.. Principles of Econometrics. Santa Barbara, Wiley Hamilton Publication, 1971. 